\title{
Pneumocephalus Following an Accidental Dural Puncture, Treated Using Hyperbaric Oxygen Therapy. A Case Report
}

\author{
Flávio Marino ${ }^{1 *}$, André Ferreira Simões ${ }^{1}$, Ângela Simas ${ }^{1}$, João Gonçalves Pereira ${ }^{1,2,3}$ \\ 1 Intensive Care Unit Department, Hospital Vila Franca de Xira, Vila Franca de Xira, Lisboa, Portugal \\ 2 Nova Medical School, Universidade Nova de Lisboa, Lisboa, Portugal \\ 3 Grupo de Infeção e Sépsis, Porto, Portugal
}

\begin{abstract}
Introduction: Neuraxial techniques, including epidural anaesthesia, are often used for perioperative pain control and are generally safe. However, both transient, mild and even severe, life-threatening neurologic complications can occur. Case presentation: A seventy-eight-year-old man was admitted to the hospital for a radical nephrectomy plus transurethral resection due to kidney and bladder cancer. During the epidural exploration, an accidental dural puncture was noted. This was followed by the patient complaining of an intense headache. The epidural catheter was placed in a different location, and surgery was performed uneventfully. The patient presented with confusion, agitation, vertical nystagmus, vision loss, and paraparesis about two hours later. The epidural levobupivacaine and morphine infusion were stopped, followed by motor block resolution. A computerized head-tomography scan showed extra-axial multiple air spots involving the frontal and temporal lobes. Emergent hyperbaric oxygen therapy was commenced. After a single session, there was complete resolution of all symptoms and a marked reduction in the number and volume of the extra-axial air visible on the CT scan. Conclusions: Although rare, pneumocephalus is a well-recognized complication of a dural puncture. Its rapid recognition in a patient with new-onset neurological symptoms and early treatment with hyperbaric oxygen therapy allows rapid clinical and imaging resolution and an improved prognosis.
\end{abstract}

Keywords: pneumocephalus, accidental dural puncture, hyperbaric oxygen therapy

Received: 11 December 2020 / Accepted: 25 May 2021

\section{INTRODUCTION}

The use of neuroaxis procedures, either as an anaesthetic or perioperative pain control, is generally safe. However, an increase in its use has led to a rise in the number of complications reported [1], namely local haemorrhage or infection, subcutaneous emphysema, venous embolism, spinal cord or nerve root compression, cardiac arrest and pneumocephalus [2].

Pneumocephalus refers to air in the intracranial cavity, either in the epidural, subdural, subarachnoid, and intraventricular or intraparenchymal space [3]. Trauma, both head and facial, is the most common cause, although neoplasms, central nervous system infections, congenital anatomical defects, barotrauma, positive pressure ventilation, surgical or anaesthetic procedures have also been reported [4].
Pneumocephalus is a rare complication of epidural catheterization, usually resulting in the accidental injection of air into the intrathecal space [1,5]. Although controversial, this epidural "air technique" is generally regarded as safer in case of an accidental dural puncture, though it may be associated with a higher rate of other complications [1,2].

The clinical presentation of pneumocephalus is nonspecific and may overlap the post-dural puncture headache or opioid intoxication. The most common symptom is severe post-puncture headache, commonly associated with confusion, vomiting, nausea, dizziness, seizures, motor weakness or focal neurological symptoms [6]. As little as two $\mathrm{mL}$ of air can be symptomatic. Clinical severity depends on the distribution and the amount of intracranial air [1]. Pneumocephalus is easily diagnosed with a computerized head-tomography 
(CT) scan, which can detect an amount of air as low as $0.5 \mathrm{~mL}$ [7].

Treatment depends on the severity of clinical presentation. Typically, complete reabsorption of the air occurs after 2-5 days [8]. Bed rest, head elevation, oxygen therapy, avoidance of Valsalva manoeuvres and cough is recommended [4]. In the more severe cases, hyperbaric oxygen therapy, although controversial, can be beneficial $[3,5,9]$, especially in highly symptomatic patients, with multiple air bubbles on imaging or with hemodynamic compromise. The high concentration of provided oxygen replaces the nitrogen in the air bubbles, allowing its reabsorption [3]. Despite the small number of cases reported and the absence of clear recommendations, hyperbaric oxygen therapy appears to be associated with faster symptoms relief and clinical improvement.

\section{CASE PRESENTATION}

A seventy-eight-year-old male was admitted to the Hospital Vila Franca de Xira, a community general hospital in Vila Franca de Xira, Portugal, for a general urological procedure. An abdominal CT scan performed eight days previously showed a large exophytic left kidney mass and diffuse thickening of the bladder wall, highly suggestive of malignancy. His past medical history indicated that he had hypertension, paroxysmal atrial fibrillation, cerebrovascular disease, ischemic coronary artery disease with congestive heart failure, dyslipidaemia, smoking-related chronic obstructive pulmonary disease, and chronic inflammatory anaemia.

A left radical nephrectomy with transurethral resection of the bladder (TUR-B) was performed. On the second day post-admission, a left radical nephrectomy with transurethral resection of the bladder (TUR-B) was performed. The surgical procedure lasted three hours under combined general anaesthesia, induced with propofol (Baxter, Utrecht, Netherlands), fentanyl (Labesfal, Tondela, Portugal) and rocuronium (Hikma, Terrugem, Portugal), and maintained with sevoflurane gas (Baxter, Lessines, Belgium) and intrathecal levobupivacaine (Fresenius Kabi, Tondela, Portugal) plus morphine (B Braun, Jaen, Spain) bolus. This was well tolerated despite significant blood loss, with the haemoglobin concentration decreasing from $10.7 \mathrm{~g} / \mathrm{dL}$ to $6.6 \mathrm{~g} / \mathrm{dL}$ during the surgery.

The epidural catheterization was performed at the L3-L4 level, using the "saline search" technique. Dur- ing this procedure, an accidental dural puncture, $2.5 \mathrm{~cm}$ in length, occurred.

The patient immediately reported having a headache; this was resolved with the removal of the catheter. A second L4-L5 epidural catheter, six cm in length, was uneventfully placed $3.5 \mathrm{~cm}$ inside the epidural space, using the loss of resistance "air" technique. Intrathecal boluses of $15 \mathrm{mg}$ of levobupivacaine (Fresenius Kabi, Tondela, Portugal) and 2mg morphine (B Braun, Jaen, Spain) were given one hour after the beginning of the surgery without any further complication.

The patient was admitted to the intensive care unit, immediately postoperative and two days after admission to the hospital.

On admission to the intensive care unit, he was drowsy due to residual sedation. On physical examination, no motor deficits were noted. Epidural levobupivacaine (Fresenius Kabi, Tondela, Portugal) and morphine (B Braun, Jaen, Spain) infusion were started for pain control. About two hours later, the patient became confused and agitated. Vertical nystagmus, vision loss and paraparesis, more prominent on the left side, were noted. The epidural infusion was immediately stopped, resulting in the resolution of the motor block.

Due to the previous unintended dural puncture, a head CT scan was immediately performed. This showed multiple extra-axial air bubbles involving both hemispheres' frontal and temporal lobes. (Figure 1).

Despite three hours having elapsed since the end of the surgery, significant neurological deterioration was still present, with no evidence of spontaneous clinical improvement. Arrangement for an immediate eighthour hyperbaric oxygen therapy session, with a 2.8 atmospheres pressure, equivalent to a depth of 18 meters, in the Lisbon Hyperbaric Medicine Center was made.

A hyperbaric oxygen therapy session was started seven hours after the end of the surgery. The treatment was well-tolerated, and no complications were reported. Significant clinical improvement was noted only four hours after starting this treatment, with complete resolution of the neurological manifestations. The patient returned to the intensive care unit at Hospital Vila Franca de Xira after the hyperbaric oxygen treatment.

A second head CT scan was performed on the second postoperative day, five hours after the end of the hyperbaric oxygen therapy session, showed a significant reduction in the air bubble volume (Figure 2), with only minor residual air foci in the right anterior 

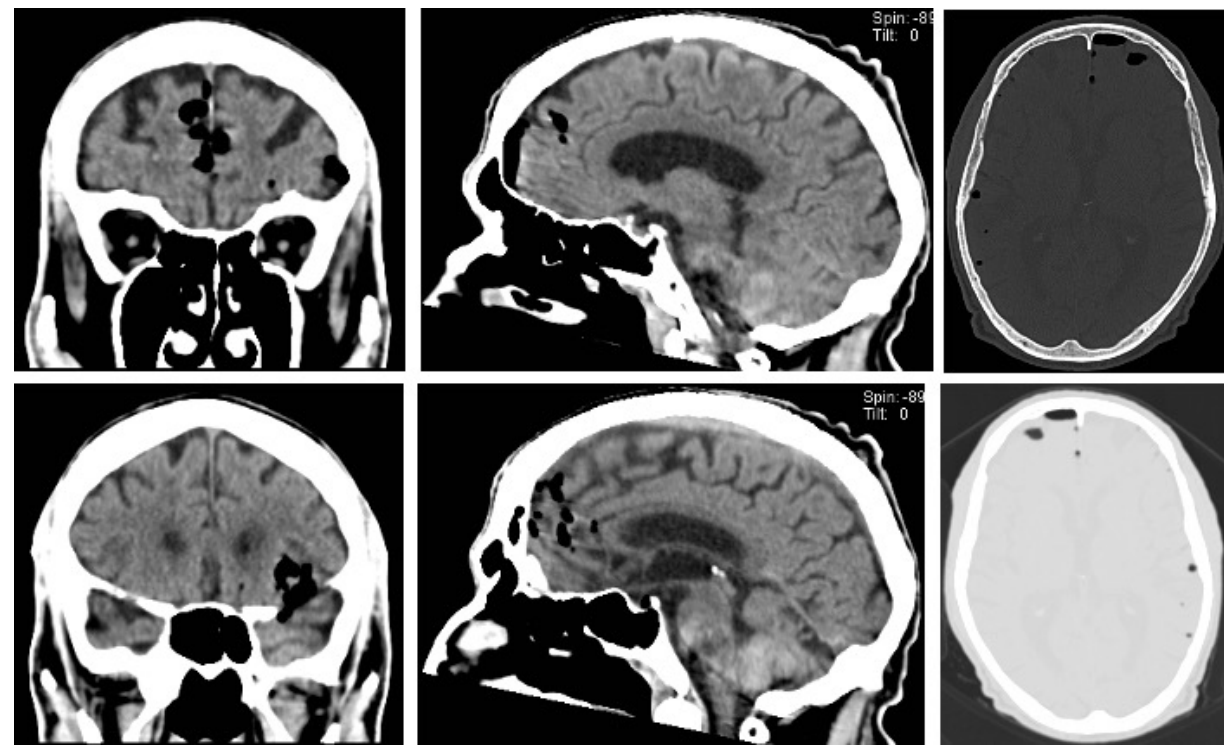

Fig. 1. Cranioencephalic computerized tomography after the surgery: the presence of several small gas foci scattered in an extra-axial location involving the frontal and temporal region of both hemispheres (with a slight left predominance).
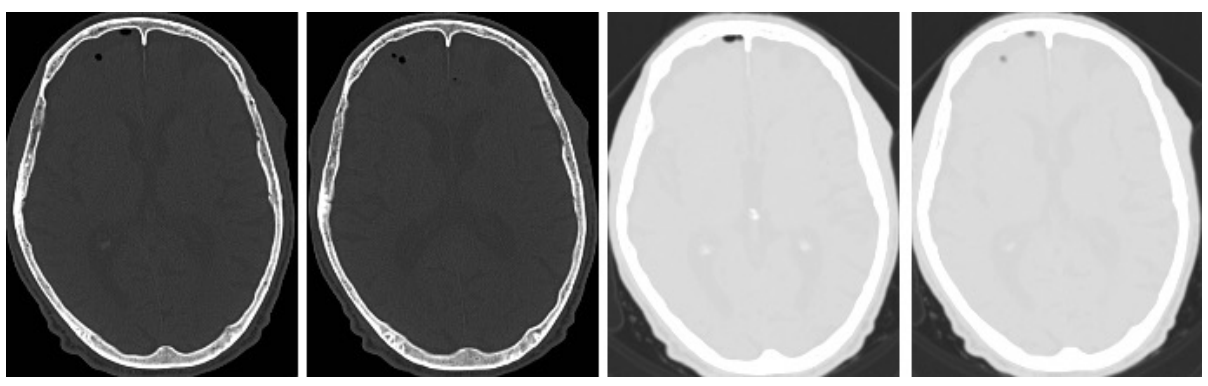

Fig. 2. Cranioencephalic computerized tomography after the hyperbaric oxygen therapy: reduction in the total number and volume of intracranial air bubbles. Small air foci persisted in the right anterior subdural space, left frontal and temporal cortical grooves.

subdural space, in the left frontal and temporal cortical grooves. No further hyperbaric oxygen therapy was needed.

The patient was transferred to the urology ward on the fifth hospital day and was discharged on the following day.

\section{Discussion}

A clinical case study is reported of an iatrogenic pneumocephalus resulting from air injected into the epidural space during a common anaesthetic technique. Significant and persistent neurological disability was noted that quickly resolved after hyperbaric oxygen therapy treatment.

Pneumocephalus is rare but may occur as a complication of the "air technique" for epidural catheter placement $[1,5,6]$. Even small amounts of air $(<2 \mathrm{~mL})$ may lead to pneumocephalus, with disabling neuro- logical symptoms, as shown in this case. It has been suggested that the "air technique" should be replaced by a "saline technique" [2]. In our patient, the "air technique" was performed as a rescue procedure after an asymptomatic complication of the "saline search" due to the unusual short depth of the dural space to prevent another accidental dural puncture.

It is hypothesized that this small amount of air could have resulted in a progressive dissection of the neurologic fibres and worsening of the neurological and cognitive manifestations. The clinical picture may overlap a common post-operatory evolution, with drowsiness or delirium triggered by anaesthetic drugs or opioid analgesia and motor block complicating epidural analgesia. Consequently, we believe that it is imperative to maintain a high level of suspicion when any air is injected into any layer of the neuroaxis.

Headaches may complicate dural puncture in as much as $40-80 \%$ of episodes $[5,10]$ and should be re- 
garded as an earlier warning sign during postoperative care. In our patient, an early headache, along with worsening neurological deterioration that occurred after a neuroaxis approach, lead to a high suspicion of pneumocephalus. A head CT scan confirmed the diagnosis.

Early recognition allowed an early referral for hyperbaric oxygen therapy. Although controversial, hyperbaric oxygen therapy appears to accelerate air "wash out", leading to faster neurological recovery and preventing further deterioration, as we reported in this case. Its rationale is based on Boyle's Law, the reduction in inert gases volume and diameter, a consequence of the high pressure, creates a gradient that promotes oxygen diffusion and nitrogen replacement. Consequently, hyperbaric oxygen therapy promotes a more rapid exchange of nitrogen with oxygen, which is more soluble, and quickly reabsorbed [3].

The hyperbaric oxygen therapy treatment duration should be guided by clinical and radiographic evolution. In our case, only one treatment was needed to resolve the whole clinical picture, in line with previous reports $[5,9]$.

Of note, the hyperbaric oxygen therapy treatment may also be complicated by toxicity. Common clinical manifestations are middle ear barotrauma, nausea, confusion, myopia and, rarely, seizures [11]. Its prevention relies mainly on ensuring large enough intervals between treatments or periodically reducing oxygen supplementation during the hyperbaric oxygen therapy session. Transferring a critically ill patient to a hyperbaric chamber may not be straightforward; careful evaluation of the patient's situation should be given priority.

We concur that hyperbaric oxygen therapy should be considered in severe cases of pneumocephalus, especially in patients with progressive and persistent symptoms, promoting faster resolution of pneumocephalus and a shorter ICU and hospital length of stay [3].

\section{CONCLUSION}

Pneumocephalus is a rare complication of epidural catheterization with the "air technique". It may lead to devastating neurological consequences, and therefore, in the appropriate setting, a high level of suspicion is imperative. Early hyperbaric oxygen therapy could improve clinical recovery, better prognosis, and a shorter stay in both hospital and intensive care units.

\section{ACKNOWLEDGMENTS}

All staff of Centro de Medicina Subaquática e Hiperbárica da Marinha, Lisboa, Portugal.

Raquel Veiga, MD, Anesthesiology Department, Hospital Vila Franca de Xira, Vila Franca de Xira, Lisboa, Portugal.

\section{- CONFLICT OF INTEREST}

None to declare

\section{REFERENCES}

1. Singh S, Thakur H. Pneumocephalus after lumbar epidural. Med J Armed Forces India. 2018;74:397-9. http://dx.doi. org/10.1016/j.mjafi.2017.09.011

2. Chew YW, Suppan VKALL, Ashutosh SR, Tew MM, Jimmy-Tan $\mathrm{JH}$. Pneumocephalus following combined spinal-epidural anaesthesia for total knee arthroplasty: A case report. Malaysian Orthop J. 2017;11:42-4.

3. Paiva WS, de Andrade AF, Figueiredo EG, Amorim RL, Prudente M, Teixeira MJ. Effects of hyperbaric oxygenation therapy on symptomatic pneumocephalus. Ther Clin Risk Manag. 2014;10:769-73.

4. Dabdoub C, Salas G, Silveira E doN, Dabdoub C. Review of the management of pneumocephalus. Surg Neurol Int. 2015;6:155.

5. Shih CC, Tsai SH, Liao WI, Wang JC, Hsu CW. Successful treatment of epidural anesthesia-induced severe pneumocephalus by hyperbaric oxygen therapy. Am J Emerg Med. 2015;33:1116. e1-1116.e3.

6. Sweni S, Senthilkumaran S, Balamurugan N, Thirumalaikolundusubramanian P. Tension pneumocephalus: A case report with review of literature. Emerg Radiol. 2013;20:573-8.

7. Rao V, Fredriksli O, Gulati S. Post-traumatic epidural tension pneumocephalus: A case report. J Med Case Rep. 2015;9:1-4.

8. Gómez-Ríos M, Fernández-Goti MC. Pneumocephalus after Inadvertent Dural Puncture during Epidural Anesthesia. Anesthesiology. 2013;118:444.

9. Lin CH, Lin SM, Lan TY, Pao JL. Pneumocephalus with Conscious Disturbance After Full Endoscopic Lumbar Diskectomy. World Neurosurg. 2019;131:112-5. https://doi.org/10.1016/j. wneu.2019.07.200

10. Barbosa FT, Cunha RM, Rocha APC, Junior HJLS. Intraventricular Pneumocephalus after Accidental Perforation of the Dura Mater. Case Report. Rev Bras Anestesiol. 2006;56:511-7.

11 Camporesi EM. Side effects of hyperbaric oxygen therapy. Undersea Hyperb Med. 2014;41:253-7. 